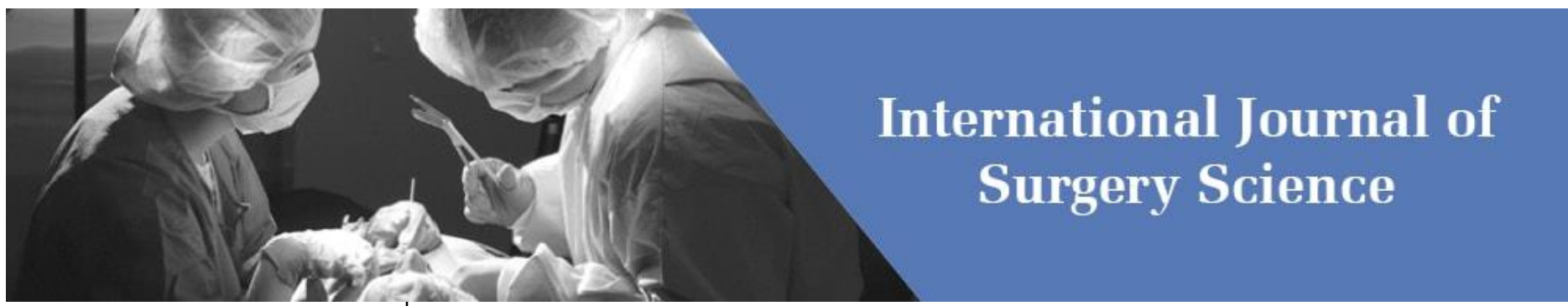

E-ISSN: 2616-3470

P-ISSN: 2616-3462

(C) Surgery Science

www.surgeryscience.com

2019; 3(4): 388-390

Received: 13-08-2019

Accepted: 15-09-2019

Dr. Nikhil Singh

Associate Professor, Department of

General Surgery Government

Medical College, Kannauj, Uttar

Pradesh, India
Corresponding Author: Dr. Nikhil Singh Associate Professor, Department of General Surgery Government Medical College, Kannauj, Uttar Pradesh, India

\section{Evaluation of complications associated with thyroid surgery in patients}

\section{Dr. Nikhil Singh}

DOI: https://doi.org/10.33545/surgery.2019.v3.i4g.274

Abstract

Background: Thyroid disorders are one of the most common endocrine diseases. The present study assessed complications associated with thyroid surgery in patients.

Materials \& Methods: This retrospective study was conducted on 54 patients in which thyroidectomy was performed in last 4 years of both genders. Preoperative investigations such as $\mathrm{T}_{3}, \mathrm{~T}_{4}, \mathrm{TSH}, \mathrm{FNAC}$, ultrasonography of thyroid gland were performed. CT scan was done where indicated. Complications of surgery were also noted.

Results: Out of 54 patients, males were 20 and females were 34. Common thyroid diseases were solitary nodular goiter in 21, multi nodular goiter in 13, adenomatous goiter in 12 and follicular carcinoma in 8 . The difference was significant $(P<0.05)$. Common complications were air way obstruction in 3 , hemorrhage in 4, hypoparathyroidism in 8 , wound infection in 2 , thyrotoxic crisis in 5 and recurrent laryngeal nerve paralysis in 5 cases. The difference was significant $(P<0.05)$.

Conclusion: Authors found that common complications are air way obstruction, hemorrhage, hypoparathyroidism, wound infection, thyrotoxic crisis and recurrent laryngeal nerve paralysis.

Keywords: Thyroid disorders, neoplastic, inflammatory

\section{Introduction}

Thyroid disorders are one of the most common endocrine diseases. Neoplastic, inflammatory and endocrine abnormalities of the thyroid gland are extremely common affecting approximately $11 \%$ of the general population ${ }^{[1]}$. Surgical resection of the thyroid gland may be necessary for the treatment of these disorders. Thyroidectomy is recommended for benign condition such as symptomatic large goiters and for the treatment of malignant disease of the thyroid gland. Thyroid surgeries are the most common endocrine surgeries performed now a day. This procedure has tremendous evolution to make it a safe surgery. In spite of improved techniques, every thyroid surgeon has come across complications associated with this surgery ${ }^{[2]}$.

Following thyroidectomy complications may develop, these are immediate and late complications, such as hemorrhage, dyspnoea, seroma, haematoma, recurrent laryngeal nerve paralysis, thyroid crisis, wound infection and hypoparathyroidism. The duration of hospital stay, following surgical procedures, has decreased significantly in recent years. Traditionally, patients undergoing thyroid surgery are observed for up to $72 \mathrm{~h}$ before discharge. During the last 10 years, short-stay thyroid surgery $(<24 \mathrm{~h}$ hospital stay) has been performed and reported in the literature as safe and cost-effective. Bilateral paralysis leads to severe airway obstruction necessitating an urgent tracheostomy in the majority of patients ${ }^{[3]}$. The present study assessed complications associated with thyroid surgery in patients.

\section{Materials \& Methods}

This retrospective study was conducted in department of general surgery. It comprised of 54 patients in which thyroidectomy was performed in last 4 years of both genders. Patients were informed regarding the study and written consent was taken. Ethical approval was obtained prior to the study.

Patient information such as name, age, gender etc. was recorded. Preoperative investigations such as T3, T4, TSH, FNAC, ultrasonography of thyroid gland were performed. CT scan was done where indicated. Complications of surgery were also noted. Results thus obtained were subjected to statistical analysis using chi-square test. $\mathrm{P}$ value $<0.05$ was considered significant. 
Results

Table 1: Distribution of patients

\begin{tabular}{|c|c|c|}
\hline \multicolumn{3}{|c|}{ Total- 54 } \\
\hline Gender & Males & Females \\
\hline Number & 20 & 34 \\
\hline
\end{tabular}

Table I shows that out of 54 patients, males were 20 and females were 34.
Table 2: Type of thyroid diseases

\begin{tabular}{|c|c|c|}
\hline Thyroid diseases & Number & P value \\
\hline Solitary nodular goiter & 21 & \multirow{2}{*}{0.05} \\
\cline { 1 - 2 } Multi nodular goiter & 13 & \\
\cline { 1 - 2 } Adenomatous goiter & 12 & \\
\cline { 1 - 2 } Follicular carcinoma & 8 & \\
\hline
\end{tabular}

Table II, graph I shows that common thyroid diseases were solitary nodular goiter in 21 , multi nodular goiter in 13 , adenomatous goiter in 12 and follicular carcinoma in 8. The difference was significant $(P<0.05)$.

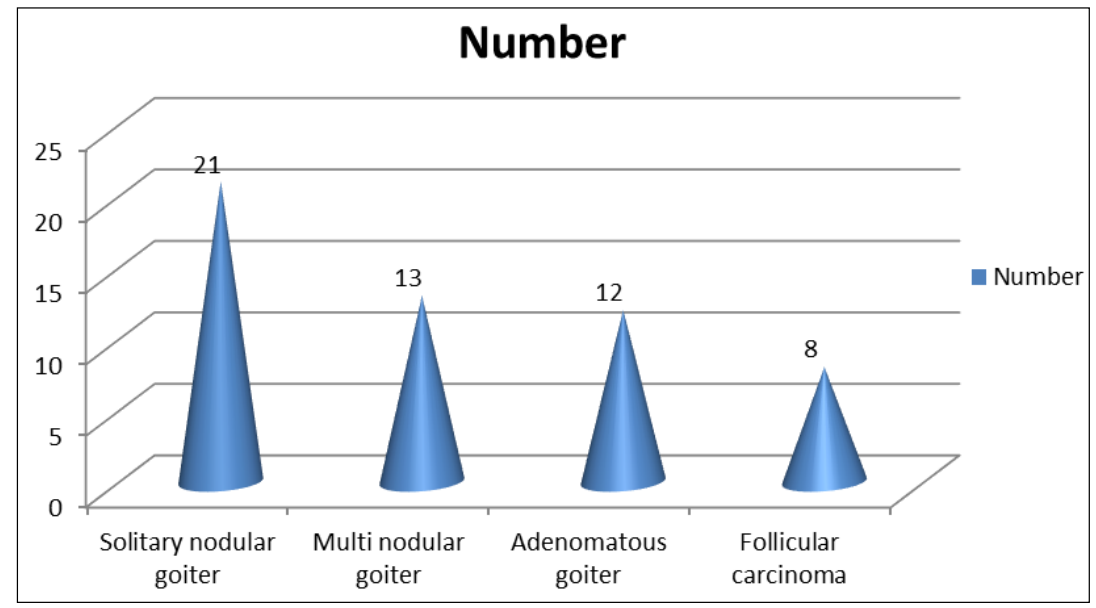

Graph 1: Type of thyroid diseases

Table 3: Complications of thyroid surgery

\begin{tabular}{|c|c|c|}
\hline Complications & Number & P value \\
\hline Air way obstruction & 3 & \\
\hline Hemorrhage & 4 & \multirow{2}{*}{0.03} \\
\hline Hypoparathyroidism & 8 & \\
\hline Wound infection & 2 & \\
\hline Thyrotoxic crisis & 5 & \\
\hline Recurrent laryngeal nerve paralysis & 5 & \\
\hline
\end{tabular}

Table III, graph II shows that common complications were air way obstruction in 3 , hemorrhage in 4 , hypoparathyroidism in 8 , wound infection in 2 , thyrotoxic crisis in 5 and recurrent laryngeal nerve paralysis in 5 cases. The difference was significant $(P<0.05)$.

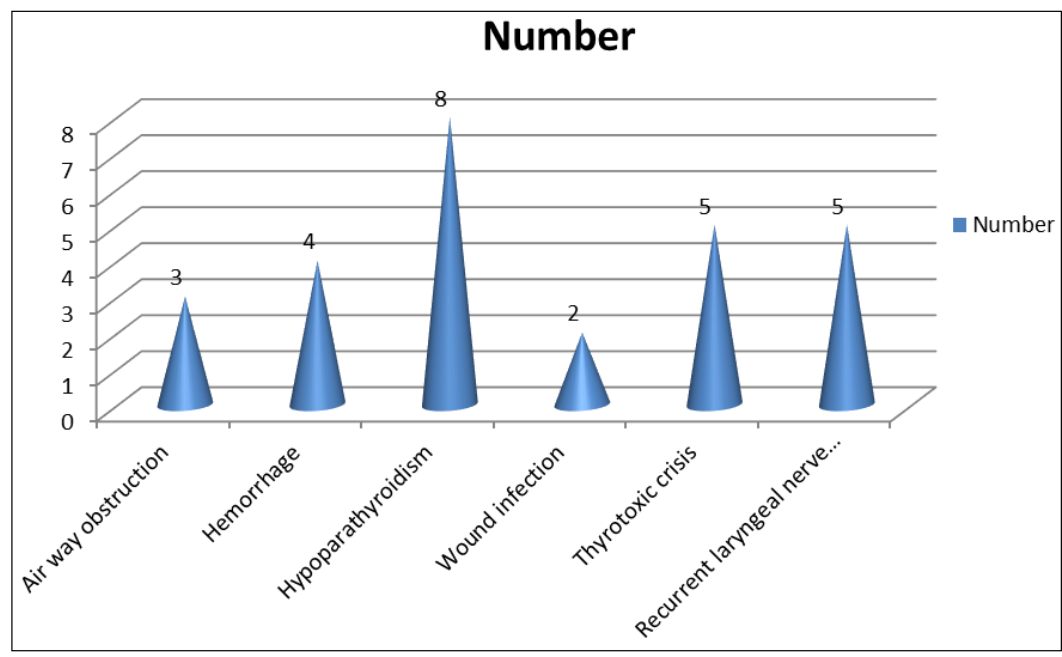

Graph 2: Complications of thyroid surgery

\section{Discussion}

Thyroidectomy has potential complications. The complications associated with thyroid surgery occur both with inpatient and outpatient surgery, but the time frame for monitoring is reduced in the outpatient setting ${ }^{[4]}$. This time frame focuses special attention on hypocalcaemia and formation of hematoma. 
Significant morbidity, following thyroidectomy is uncommon, and occurs in less than $6 \%$ of cases. The most serious complication related to thyroid surgery is post-operative hemorrhage with the potential for tracheal compression, airway involvement, and death. Immediate or early hemorrhage occurs in less than $1 \%$ to $2 \%$ of cases ${ }^{[5]}$.

The major postoperative complications are hypocalcemia, wound infection, hematoma, recurrent laryngeal nerve (RLN) injury, and Horner's syndrome. Hypocalcemia is the important postoperative complication of thyroid surgery causing potentially severe symptoms and increasing hospitalization time [6]. Hypoparathyroidism is the usual cause of hypocalcemia, it results from accidental gland injury, removal, or devascularization. Hoarseness is mostly caused by RLN injury, which often results in vocal and laryngeal dysfunction. The patient's quality of life can be negatively influenced by the incidence of potential complications leading to increase in individual's health-care costs and requiring a lifelong alternative therapy. Complications associated with thyroidectomy are related to the type of disease, extent of disease, removal approaches, surgeon's training, and experience ${ }^{[7]}$. The present study assessed complications associated with thyroid surgery in patients.

In present study, out of 54 patients, males were 20 and females were 34 . Common thyroid diseases were solitary nodular goiter in 21, multi nodular goiter in 13, adenomatous goiter in 12 and follicular carcinoma in 8 . Kahky et al. ${ }^{[8]}$ found that $12 \%$ of the patients had transient hypoparathyroidism, 4\% had hemorrhage, $2 \%$ had permanent hypoparathyroidism, 4\% had temporary recurrent laryngeal nerve (RLN) palsy and $2 \%$ had permanent recurrent laryngeal nerve palsy. Other rare complications were superior laryngeal nerve palsy, hematoma, and wound infection. Improved surgical techniques and proper management of complications reduce the postoperative morbidity and mortality of thyroid surgery. Iqbal et al. [9] reviewed post-operative bleeding in patients who had undergone thyroidectomy, and they described a critical period of time in which bleeding occurs most commonly (in all cases the potential for airway compromise was identified within 4 hours of surgery).

We found that common complications were air way obstruction in 3 , hemorrhage in 4 , hypoparathyroidism in 8 , wound infection in 2, thyrotoxic crisis in 5 and recurrent laryngeal nerve paralysis in 5 cases. Kumath et al. ${ }^{[10]}$ study comprised 175 total thyroidectomies, 93 hemi-thyroidectomies and 2 isthmusectomies. No cases of death or post-operative haemorrhage occurred in any of these patients. Permanent unilateral recurrent laryngeal nerve injury was observed in 4 patients $(1.48 \%)$. Transient post-operative hypocalcaemia occurred in 23 patients, whereas permanent post-operative hypocalcaemia was observed in 8 patients $(2.96 \%) ; 4$ patients were re-admitted and required early calcium supplementation. Five patients failed to tolerate the diet during the immediate post-operative period. The average duration of hospital stay was 1.02 days. Considering the 4 patients who required re-admission due to hypocalcaemia, the total length of hospital stay was 1.05 days. Khaky et al. in his study showed that the overall complication rate for combined surgeries was $14.2 \%$ with recurrent laryngeal nerve injury more common than hypoparathyroidism.

\section{Conclusion}

Authors found that common complications are air way obstruction, hemorrhage, hypoparathyroidism, wound infection, thyrotoxic crisis and recurrent laryngeal nerve paralysis.

\section{References}

1. Mahmood S, Hossain GA, Rahman MU, Khan N, Islam SMM. Awareness of General Population regarding goitre, Mym. Med. Journal. 1995; 4(2):86-91.

2. Conaris GJ, Manowitz NR, Mayor G et al. The Colorado Thyroid Disease Prevalence study. Arch Intern Med. 2000; 160:526-534.

3. Haward Russell John. Diagnosis Proceeds Treatment In : Jk watkinson, Stell and Maran 's Head and Neek Surgery, (4th ed), 459-478.

4. Wade JSH. The morbidity of subtotal thyroidectomy, British Journal of surgery. 1960, 48-42.

5. Lalida Kasemsewan M, Shutima Nubthuenetr. Journal of Otolaryngology. 1997; 26(6):32-35.

6. Patil PU, Godhi AS, Sant AN. Fine needle aspiration cytology of papillary carcinoma thyroid with Hashimoto 's thyroiditis: Report of two cases. Indian J Pathol microbial. 1997; 40:165-8.

7. Durham CF, Harrison TS. The surgical anatomy of the superior laryngeal nerve. Surgery Gynecology and Obstetrics. 1964; 118:38-43.

8. Kahky MD, Weber RS. Complications of Surgery of the thyroid and Parathyroid glands. Surgclin NAM. 1993; 73:307-20.

9. Iqbal M, Rasool MT, Khan M, Malik S, Barkat W. Prospective study of 111 cases of thyroid surgery for postoperative morbidity and mortality. J park Med Assoc. 1989; 39:201-4.

10. Kumath M, Hussock Marushci F, Horschig Gastinger P. Identification of recurrent laryngeal nerve by infraoperative neuromonitoring. 1999; 124(7):641-5. 